\title{
Massive gravity and structure formation
}

\author{
Michael V. Bebronne ${ }^{1}$ and Peter G. Tinyakov ${ }^{2,3}$ \\ ${ }^{1}$ Service de Physique Théorique, Université Libre de Bruxelles (U.L.B.), \\ CP225, boulevard. du Triomphe, B-1050 Bruxelles, Belgium. \\ ${ }^{2}$ Service de Physique Théorique, Université Libre de Bruxelles (U.L.B.), \\ CP225, bld. du Triomphe, B-1050 Bruxelles, Belgium. \\ ${ }^{3}$ Institute for Nuclear Research of the Russian Academy of Sciences, \\ 60th October Anniversary Prospect, 7a, 117312 Moscow, Russia.
}

(Dated: October 26, 2018)

\begin{abstract}
We study the growth of cosmological perturbations in the model of Lorentz-violating massive gravity. The Friedman equation in this model acquires an unconventional term due to the Lorentzbreaking condensates which has the equation of state $w=-1 /(3 \gamma)$ with $\gamma$ being a free parameter taking values outside of the range $[0,1 / 3]$. Apart from the standard contributions, the perturbations above the Friedmann background contain an extra piece which is proportional to an arbitrary function $\vartheta\left(x^{i}\right)$ of the space coordinates. This function appears as an integration constant and corresponds to a non-propagating scalar mode which may, however, become dynamical with the account of the higher-derivative corrections. For $-1<\gamma<0$ and $\gamma=1$ the "anomalous" perturbations grow slower than the standard ones and thus the model is compatible with observations. Whether the model is experimentally acceptable at other values of $\gamma$ depends on the value of the function $\vartheta\left(x^{i}\right)$ at the beginning of the radiation-dominated epoch.
\end{abstract}

\section{INTRODUCTION}

The standard cosmological model is based on the assumption that the gravitational interaction is correctly described by the general relativity (GR) at scales comparable to the horizon size. This model is quite successful in describing the bulk of the cosmological data: the anisotropies of the cosmic microwave background radiation, the primordial abundance of light elements and the structure formation in the early Universe. The quantitative agreement between the standard cosmological model and the observations has an ever-growing precision [1]. The question arises to which extent one should consider this agreement as a confirmation of the general relativity itself.

In order to address this question an alternative model is needed whose predictions can be compared to those of GR. Such a model should coincide with GR at scales from $\sim 0.1 \mathrm{~mm}$ to the size of the solar system where GR has been tested directly. Therefore, the modifications of the gravitational interaction should occur in the infrared, at distances much larger than the size of the solar system.

Interestingly, at these distances the predictions of GR actually do not agree with the observations; only after the introduction of the otherwise undetected dark matter and dark energy the agreement is achieved. The necessity for these new components is a major problem of the standard cosmology. Hence, in parallel with the direct searches for the dark components, the alternative models of gravity should be explored which may eventually eliminate the need (or provide alternative candidates) for the dark matter and shed new light on the nature of the dark energy.

It is a challenging problem to modify the gravitational interaction at large distances. An alternative model has to be theoretically consistent, i.e., free from ghosts and instabilities. In addition, it should be in agreement with the existing experimental data and should, ideally, provide testable predictions for the future experiments. It is not obvious that such models exist, so it would be very important to construct an example.

There have been several attempts made in this direction $[2,3,4,5,6,7,8,9,10]$. In this paper we concentrate on the massive gravity model [11] which is minimal in the sense that in does not contain new light propagating degrees of freedom as compared to the Einstein gravity. In this model the graviton acquires a mass due to the space-time dependent condensates of the four "Goldstone" scalars $\phi^{0}(x), \phi^{i}(x)$. The action of the model reads [12]

$$
\mathcal{S}=\int \mathrm{d}^{4} x \sqrt{-g}\left[-M_{P l}^{2} \mathcal{R}+\Lambda^{4} \mathcal{F}\left(Z^{i j}\right)+\mathcal{L}_{\text {matter }}\right]
$$

where the first term is the standard Einstein-Hilbert action, $\mathcal{L}_{\text {matter }}$ stands for the minimally coupled ordinary matter and $\mathcal{F}\left(Z^{i j}\right)$ is a function of the derivatives of the four scalar fields $\phi^{0}(x), \phi^{i}(x)$ which depends on a single argument $Z^{i j}$ constructed as follows:

$$
\begin{aligned}
Z^{i j} & =X^{\gamma} W^{i j} \\
X & =\Lambda^{-4} g^{\mu \nu} \partial_{\mu} \phi^{0} \partial_{\nu} \phi^{0}, \\
V^{i} & =\Lambda^{-4} g^{\mu \nu} \partial_{\mu} \phi^{0} \partial_{\nu} \phi^{i}, \\
W^{i j} & =\Lambda^{-4} g^{\mu \nu} \partial_{\mu} \phi^{i} \partial_{\nu} \phi^{j}-\frac{V^{i} V^{j}}{X} .
\end{aligned}
$$

The constant $\gamma$ is a free parameter. The model possesses meaningful cosmological solutions for $\gamma \geq 1 / 3$ and $\gamma<0$ [13].

The flat space vacuum solution is [15]

$$
\phi_{0}=\Lambda^{2} t, \quad \phi^{i}=\Lambda^{2} x^{i} .
$$


The ground state is translationally invariant due to the derivative nature of the Goldstone coupling, but the Lorentz symmetry is spontaneously broken. The particular dependence of the action (1) on the derivatives of the Goldstone fields through a single argument $Z^{i j}$ ensures the non-pathological behavior of the perturbations about the vacuum solution [11], namely, the absence of ghosts and rapid instabilities. The tensor metric perturbations are, in general, massive with the mass determined by the first and the second derivatives of the function $\mathcal{F}$. The low-energy spectrum consists of the two propagating tensor modes. The auxiliary scalars do not appear in the spectrum.

An interesting and peculiar feature of this model is that the gravitational interaction between static sources is described by the standard Newton's law despite the non-zero mass of the graviton[16]. Due to this feature the model passes the terrestrial and solar system tests even for graviton masses as large as $\left(10^{15} \mathrm{~cm}\right)^{-1}$. Moreover, it admits the standard Friedmann-RobertsonWalker cosmological solutions, the only trace of the Goldstone scalars being two contributions to the energy density which behave as a cosmological constant and as matter with the equation of state $w=-(3 \gamma)^{-1}$. The massive gravitons may be created in cosmologically significant amount and may play a role of the dark matter [12].

Given that the model passes the most obvious constraints one may wonder if it reproduces correctly more subtle parts of modern cosmology, in particular, the theory of structure formation. This is the question which we address in this paper. The answer is not obvious a priori since the vacuum in this model contains the condensates of the Goldstone fields whose perturbations mix with the matter density perturbations.

We find that cosmological perturbations in the model (1) consist of two parts. The first part behaves identically to the perturbations in GR and is, therefore, compatible with observations. The second, "anomalous" part is proportional to an unknown function $\vartheta\left(x^{i}\right)$ of the space coordinates which arises as an integration constant.

The appearance of this function reflects the existence of the non-dynamical mode with the dispersion relation $\omega^{2}=0$, much in common with the ghost condensate model [9]. In the model (1) the value of $\vartheta\left(x^{i}\right)$ is determined by the initial conditions. Note, however, that the action (1) is no more than the low-energy effective action. One should expect the higher-derivative corrections to eq. (1) to be present. In general, these corrections make $\vartheta\left(x^{i}\right)$ a slowly changing dynamical variable. In this case the initial value of $\vartheta\left(x^{i}\right)$ may be determined by its evolution at the inflationary epoch.

As we show below, the growth of the "anomalous" perturbations depends on the value of $\gamma$. For $-1<\gamma<0$ they grow slower than the standard ones, so that the latter dominate. At $\gamma=1$ the anomalous contributions to perturbations cancel out. Thus, at least in these two cases the perturbations behave in a standard way and the model (1) is consistent with the structure formation in the Universe. This is the main result of the paper.

For other values of $\gamma$ (in particular, for the cosmologically interesting case $1 / 3<\gamma<1$ which corresponds to the equation of state of the condensate $-1<$ $w<-1 / 3)$ the anomalous perturbations grow at the radiation-dominated epoch, and may or may not grow faster than the standard perturbations at the matterdominated stage, depending on the particular value of $\gamma$ (see Sect. III for details). Whether this leads to a contradiction with observations depends on the function $\vartheta\left(x^{i}\right)$ at the beginning of the radiation-dominated era. To address this question one needs to consider the higherderivative corrections to the action (1). This will be done elsewhere.

The paper is organized as follows. In Sect. II we review the cosmological solutions in massive gravity. In Sect. III we calculate the growth of perturbations in the FRW background. Sect. IV contains the summary of the results and their discussion. The details of the calculations are given in the Appendix.

\section{FRW SOLUTION}

In the absence of matter the model described by the action (1) admits the standard FRW solutions [13]. The spatially-flat homogeneous and isotropic ansatz [17] for the metric and Goldstone fields reads

$$
\begin{aligned}
d s^{2} & =a^{2}(\eta)\left(d \eta^{2}-\delta_{i j} d x^{i} d x^{j}\right), \\
\phi^{0} & =\Lambda^{2} \phi(\eta), \quad \phi^{i}=\Lambda^{2} x^{i},
\end{aligned}
$$

which implies

$$
Z=\phi^{2 \gamma} / a^{2 \gamma+2},
$$

where $Z=-\delta_{i j} Z^{i j} / 3$ and where prime denotes the derivative with respect to the conformal time $\eta$. Assuming the ordinary matter is homogeneous and isotropic, the equations of motion (the Friedmann equation and the field equation for $\phi^{0}$ ) take the form

$$
\begin{aligned}
\mathcal{H}^{2} & =\frac{a^{2}}{3 M_{\mathrm{Pl}}^{2}}\left(\rho_{m}+\rho_{\phi}+\rho_{\Lambda}\right), \\
0 & =\partial_{0}\left(a^{3-1 / \gamma} Z^{1-1 / 2 \gamma} \mathcal{F}_{Z}\right),
\end{aligned}
$$

where $\mathcal{H}=a^{\prime} / a$, so that $\mathcal{H} / a$ is the Hubble constant, and $3 \mathcal{F}_{Z}=\delta^{i j} d \mathcal{F} / d Z^{i j}$. The matter energy density $\rho_{m}$ has the standard form, while the two contributions of the Goldstone fields to the energy density read

$$
\rho_{\Lambda}=-\Lambda^{4} \mathcal{F} / 2, \quad \rho_{\phi}=-3 \gamma \Lambda^{4} Z \mathcal{F}_{Z} .
$$

The first of these terms behaves like a cosmological constant, while the second contribution corresponds to matter with the equation of state $w=-1 /(3 \gamma)$. Thus, the Friedmann equation (4) reduces to the standard one, the only trace of the Goldstone field being the energy densities $\rho_{\phi}$ and $\rho_{\Lambda}$. 
For a given function $\mathcal{F}$ eq. (5) determines the dependence of the variable $Z$ on the scale factor. For $\gamma>1 / 3$ or $\gamma<0$ this equation implies

$$
Z^{1-1 / 2 \gamma} \mathcal{F}_{Z} \rightarrow 0 \text { as } a \rightarrow \infty .
$$

The case of interest (which occurs generically for an algebraic function $\mathcal{F}$ [13]) is when in this limit $Z \rightarrow Z_{0}=$ const. such that $\mathcal{F}_{Z}\left(Z_{0}\right)=0$. In this case the graviton mass remains finite at $a \rightarrow \infty$ (see Sect. B for details).

In a special case $\gamma=1 / 3$ the field equation (5) implies $Z=$ const. Then $\mathcal{F}_{Z}$ is not driven to zero by the cosmological evolution and $\rho_{\phi}$ behaves as the cosmological constant. The value of the total cosmological constant $\rho_{\phi}+\rho_{\Lambda}$ is determined by the initial conditions.

In what follows we neglect where possible the deviations from the point $Z=Z_{0}$ (note that in this point $\left.\rho_{\phi}=0\right)$. We also assume that the value of $\rho_{\Lambda}$ is of the order of the present-day cosmological constant, and thus its contribution to the Friedmann equation at the epoch of structure formation is negligible.

\section{COSMOLOGICAL PERTURBATIONS}

As in the standard analysis of the cosmological perturbations [14], it is convenient to separate space and time components. The metric perturbations can be parameterized in the following way,

$$
\begin{aligned}
\delta g_{00} & =2 a^{2} \varphi \\
\delta g_{i 0} & =a^{2}\left(v_{i}+\partial_{i} B\right), \\
\delta g_{i j} & =a^{2}\left(2 \psi \delta_{i j}-\partial_{i} F_{j}-\partial_{j} F_{i}-2 \partial_{i} \partial_{j} E+h_{i j}\right),
\end{aligned}
$$

where the vector perturbations $v_{i}$ and $F_{i}$ are transverse, while the tensor perturbation $h_{i j}$ is transverse and traceless. A similar parameterization can be used for the perturbations of the Goldstone fields,

$$
\begin{aligned}
\delta \phi^{0} & =\Lambda^{2} \xi^{0}, \\
\delta \phi^{i} & =\Lambda^{2}\left(\xi_{i}+\partial_{i} \xi\right),
\end{aligned}
$$

where $\xi_{i}$ is transverse. Finally, the perturbations of the ordinary matter are parameterized in the following way,

$$
\begin{aligned}
\delta \mathcal{T}_{\mu \nu}^{m}= & \left(\delta \rho_{m}+\delta p_{m}\right) u_{\mu} u_{\nu}-g_{\mu \nu} \delta p_{m}-p_{m} \delta g_{\mu \nu} \\
& +\left(\rho_{m}+p_{m}\right)\left(u_{\nu} \delta u_{\mu}+u_{\mu} \delta u_{\nu}\right)
\end{aligned}
$$

where $\delta \rho_{m}$ and $\delta p_{m}$ are related by the matter equation of state and the perturbations of the velocity $\delta u_{\mu}$ are expressed in terms of the scalar $\zeta$ and the transverse vector $\zeta_{i}$ as follows,

$$
\begin{aligned}
\delta u_{i} & =a\left(\zeta_{i}+\partial_{i} \zeta\right) \\
\delta u_{0} & =a \varphi
\end{aligned}
$$

Therefore, in total there is one tensor perturbation $h_{i j}$ consisting of two components, four vectors $v_{i}, F_{i}, \xi_{i}$ and $\zeta_{i}$, consisting of two components each, and 9 scalars $\varphi$,
$B, \psi, E, \xi^{0}, \xi, \zeta, \delta p_{m}$ and $\delta \rho_{m}$. One vector and two scalar perturbations are gauge degrees of freedom; they can be eliminated by imposing a gauge condition. As a consequence, there is only three gauge-invariant vector fields

$$
\varpi_{i}=v_{i}+F_{i}^{\prime}, \quad \sigma_{i}=\xi_{i}-F_{i},
$$

and $\zeta_{i}$, and seven scalar gauge-invariant fields

$$
\begin{aligned}
\Phi & =\varphi-a^{-1}\left[a\left(E^{\prime}+B\right)\right]^{\prime}, \\
\Psi & =\psi+\mathcal{H}\left(E^{\prime}+B\right), \\
\Xi & =\xi-E, \\
\mathcal{B} & =B+E^{\prime}-\xi^{0} / \phi^{\prime}, \\
\delta_{\rho} & =\left[\delta \rho_{m}-\rho_{m}^{\prime}\left(E^{\prime}+B\right)\right] / \rho_{m}, \\
\delta_{p} & =\left[\delta p_{m}-p_{m}^{\prime}\left(E^{\prime}+B\right)\right] / p_{m}, \\
\delta_{\zeta} & =\zeta-\left(E^{\prime}+B\right) .
\end{aligned}
$$

The tensor perturbation $h_{i j}$ is also gauge invariant.

\section{A. The tensor perturbations}

The equation for the tensor perturbation is

$$
h_{i j}^{\prime \prime}+2 \mathcal{H} h_{i j}^{\prime}-\partial_{i}^{2} h_{i j}+a^{2} m_{2}^{2} h_{i j}=0,
$$

where $m_{2}$ is the graviton mass which has the scale $m_{2} \propto \Lambda^{2} / M_{\mathrm{Pl}}$ and whose precise expression in terms of the function $\mathcal{F}\left(Z^{i j}\right)$ and its derivatives is given in the Appendix B.

This equation is identical to the equation for a free massive scalar field in the FRW background. If the mass of the graviton is larger than the Hubble constant, $m_{2} \gg$ $\mathcal{H} / a$, which we assume to be the case in what follows, eq. (11) describes massive gravitational waves with the amplitude scaling like $h_{i j} \propto a^{-1}$ and $h_{i j} \propto a^{-3 / 2}$ in the relativistic and non-relativistic limits, respectively.

\section{B. The vector perturbations}

In the longitudinal gauge the three equations describing vector perturbations are

$$
\begin{aligned}
& \partial_{j}^{2} \varpi_{i}-2 a^{2} \rho_{m} M_{p l}^{-2}(1+w) \zeta_{i}=0, \\
& \varpi_{i}^{\prime}+2 \mathcal{H} \varpi_{i}-a^{2} m_{2}^{2} \sigma_{i}=0, \\
& m_{2}^{2} \partial_{j}^{2} \sigma_{i}=0,
\end{aligned}
$$

where $w$ is the parameter entering the equation of state of the ordinary matter, $p_{m}=w \rho_{m}$.

The first of these equations allows to express $\zeta^{i}$ in terms of $\varpi_{i}$, while the third equation gives $\sigma_{i}=0$. Therefore, the only non-trivial equation is eq. (13). It differs from the conventional one by the term proportional to the graviton mass $m_{2}^{2}$ which cancels at $\sigma_{i}=0$. Thus, this equation is the conventional one and describes a field with the amplitude decreasing as $\varpi_{i} \propto a^{-2}$. 


\section{The scalar perturbations}

The perturbation $\delta_{p}$ can be expressed in terms of $\delta_{\rho}$ by means of the matter equation of state. In the case of the adiabatic perturbations one has

$$
\delta_{p}=\frac{c_{s}^{2}}{w} \delta_{\rho},
$$

where $c_{s}$ is the sound velocity $\left(c_{s}^{2}=w\right.$ for the ideal fluid). The behavior of the remaining six perturbations is governed by the following equations,

$$
\begin{aligned}
0= & \Phi-\Psi+a^{2} m_{2}^{2} \Xi \\
0= & 2\left(\Psi^{\prime}+\mathcal{H} \Phi\right)-a^{2} \frac{\rho_{m}}{M_{P l}^{2}}\left(1+w_{\omega}\right) \delta_{\zeta} \\
0= & -2 \partial_{j}^{2} \Psi+6 \mathcal{H}\left(\mathcal{H} \Phi+\Psi^{\prime}\right)+a^{2} m_{4}^{2}\left(\partial_{j}^{2} \Xi+3 \Psi\right) \\
& +a^{2} \frac{\rho_{m}}{M_{P l}^{2}} \delta_{\rho}-a^{2} m_{0}^{2}\left[\frac{\phi^{\prime \prime}}{\phi^{\prime}} \mathcal{B}+\mathcal{B}^{\prime}+\Phi\right] \\
0= & -2 \Psi^{\prime \prime}-2 \Phi\left(\mathcal{H}^{2}+2 \mathcal{H}^{\prime}\right)+\partial_{j}^{2}(\Psi-\Phi) \\
& -2 \mathcal{H}(2 \Psi+\Phi)^{\prime}+a^{2}\left[\frac{p_{m}}{M_{P l}^{2}} \delta_{p}-m_{3}^{2} \partial_{j}^{2} \Xi\right. \\
& \left.+m_{4}^{2}\left(\Phi+\frac{\phi^{\prime \prime}}{\phi^{\prime}} \mathcal{B}+\mathcal{B}^{\prime}-\Psi / \gamma\right)\right] \\
0 & \partial_{0}\left[\frac { a ^ { 4 } m _ { 4 } ^ { 2 } } { \phi ^ { \prime } } \left(3 \gamma\left(\Phi+\mathcal{B}^{\prime}+\frac{\phi^{\prime \prime}}{\phi^{\prime}} \mathcal{B}\right)\right.\right. \\
& \left.\left.-\left(3 \Psi+\partial_{i}^{2} \Xi\right)\right)\right] \\
0= & m_{4}^{2}\left(\Psi / \gamma-\frac{\phi^{\prime \prime}}{\phi^{\prime}} \mathcal{B}-\mathcal{B}^{\prime}-\Phi\right) \\
& +\left(m_{3}^{2}-m_{2}^{2}\right) \partial_{j}^{2} \Xi .
\end{aligned}
$$

Here $m_{i}$ are the graviton mass parameters defined in the Appendix B. We assume that they are of the order of $\Lambda^{2} / M_{\mathrm{Pl}}$.

This system of equations can be solved as follows. At $m_{4}^{2} \neq 0$ eq. (20) can be used to express $\phi^{\prime \prime} / \phi^{\prime} \mathcal{B}+\mathcal{B}^{\prime}+\Phi$. Then eq. (19) becomes a closed equation for $\Xi$

$$
0=\partial_{i}^{2}\left[\Xi^{\prime}+\left(3-\frac{1}{\gamma}\right) \mathcal{H} \Xi\right]
$$

The solution of this equation which does not grow at spatial infinity reads

$$
m_{2}^{2} \Xi=-\vartheta\left(x^{i}\right) a^{1 / \gamma-3}
$$

where $\vartheta\left(x^{i}\right)$ is an integration constant depending only on spatial coordinates. Then eqs. (16), (17) and (20) can be used to express $\delta_{\zeta}, \delta_{\rho}$ and $\mathcal{B}$ in terms of $\Phi$ and $\Psi$ while eq. (15) reads

$$
\Phi-\Psi=\vartheta\left(x^{i}\right) a^{1 / \gamma-1}
$$

With the account of all these relations, the remaining eq. (18) becomes a closed inhomogeneous equation for $\Psi$

$$
\begin{aligned}
0= & \partial_{a}^{2} \Psi+\frac{1}{a}\left(4+3 c_{s}^{2}+\frac{\mathcal{H}^{\prime}}{\mathcal{H}^{2}}\right) \partial_{a} \Psi+\frac{1}{a^{2}}\left[\left(1+3 c_{s}^{2}\right)+2 \frac{\mathcal{H}^{\prime}}{\mathcal{H}^{2}}-\frac{c_{s}^{2} \partial_{i}^{2}}{\mathcal{H}^{2}}\right] \Psi \\
& -\left[\frac{\gamma c_{s}^{2} \partial_{i}^{2}}{\mathcal{H}^{2}}-\left(3 c_{s}^{2}+\frac{1}{\gamma}+2 \frac{\mathcal{H}^{\prime}}{\mathcal{H}^{2}}\right)\right] \vartheta a^{1 / \gamma-3} .
\end{aligned}
$$

Once the solution to this equation is found, the other variables are determined by eqs. (22), (16), (17) and (20). In particular, one finds

$$
\begin{aligned}
\delta_{\rho}= & \frac{2 M_{p l}^{2}}{\rho_{m}}\left(\gamma \partial_{i}^{2}-3 \mathcal{H}^{2}\right) a^{1 / \gamma-3} \vartheta \\
& -\frac{2 M_{p l}^{2}}{a^{2} \rho_{m}}\left[3 \mathcal{H}^{2}\left(1+a \frac{\partial}{\partial a}\right)-\partial_{i}^{2}\right] \Psi,
\end{aligned}
$$

where $\Psi$ is a solution to eq. (23).

The conventional cosmological perturbations are recovered by setting the graviton masses to zero, $m_{i}^{2}=0$.
In this case eq. (15) gives $\Phi-\Psi=0$ which implies $\vartheta\left(x^{i}\right)=0$ (cf. eqs. (21) and (22)). Then both eq. (23) and eq. (24) reduce to the standard equations describing cosmological perturbations in the Einstein theory. Note that the value of $\vartheta$ is determined essentially by the initial conditions. Setting $\vartheta=0$ would eliminate the $\vartheta$-dependent terms in eqs. (23) and (24) and bring these equations to the conventional form even in the case $m_{2}^{2} \neq 0$.

In the case of matter perturbations in a matterdominated Universe eq. (23) reduces to the following 
equation,

$$
\frac{\partial^{2} \Psi}{\partial a^{2}}+\frac{7}{2 a} \frac{\partial \Psi}{\partial a}+\left(\frac{1}{\gamma}-1\right) a^{1 / \gamma-3} \vartheta=0,
$$

which differs from the standard case by the presence of the inhomogeneous term proportional to $\vartheta$. The solution to this equation reads

$$
\Psi=-\frac{2 \gamma}{2+3 \gamma} a^{1 / \gamma-1} \vartheta\left(x^{i}\right)+a^{-5 / 2} c_{1}\left(x^{i}\right)+c_{2}\left(x^{i}\right),
$$

where $c_{i}\left(x^{i}\right)$ are the integration constants. Substituting this solution into eq. (24) one finds the density contrast

$$
\begin{aligned}
\delta_{\rho}= & \left(\frac{2 M_{p l}^{2} a}{\rho_{0}} \partial_{i}^{2}+3\right) \frac{c_{1}\left(x^{i}\right)}{a^{5 / 2}}+2\left(\frac{a M_{p l}^{2}}{\rho_{0}} \partial_{i}^{2}-1\right) c_{2}\left(x^{i}\right) \\
& +\frac{6 \gamma}{2+3 \gamma} a^{1 / \gamma-1}\left(\frac{a \gamma M_{p l}^{2}}{\rho_{0}} \partial_{i}^{2}-1\right) \vartheta\left(x^{i}\right) .
\end{aligned}
$$

where $\rho_{0}$ is the energy density of matter at present. The first two terms in this equation are precisely the ones which appear in the standard Einstein theory, the second term describing the linear growth of the perturbations, $\delta_{\rho} \propto a$. The difference with the conventional case consists in the third term on the r.h.s of eq. (25). The perturbations corresponding to this term grow proportionally to $a^{1 / \gamma}$. For $\gamma>1$ or $\gamma<0$ these "anomalous" perturbations grow slower than the standard ones.

In the radiation epoch the situation is similar. For a relativistic fluid one has $w_{m}=1 / 3$, so that eq. (23) reduces to the following one,

$$
\begin{aligned}
0= & \frac{\partial^{2} \Psi}{\partial a^{2}}+\frac{4}{a} \frac{\partial \Psi}{\partial a}-\frac{M_{p l}^{2} \partial_{i}^{2}}{\rho_{r}} \Psi \\
& +\left(\frac{1}{\gamma}-1-\frac{a^{2} \gamma M_{p l}^{2} \partial_{i}^{2}}{\rho_{r}}\right) a^{1 / \gamma-3} \vartheta
\end{aligned}
$$

where $\rho_{r}$ is the energy density of radiation at present. For the generic value of $\gamma$ the solution to this equation is cumbersome. For simplicity let us concentrate on the modes which are much smaller than the Hubble scale, $k^{2} \gg \mathcal{H}^{2}$. The density contrast calculated according to eq. (24) has the standard oscillating piece and the extra part proportional to $\vartheta$,

$$
\begin{aligned}
\delta_{\rho} \sim & c_{1}\left(x^{i}\right) \sin y+c_{2}\left(x^{i}\right) \cos y+2 \gamma\left(\frac{\rho_{r}}{k^{2} M_{p l}^{2}}\right)^{(1 / \gamma-1) / 2} \\
& \times\left[-y^{1+1 / \gamma}+\int_{0}^{y} \mathrm{~d} x x^{1+1 / \gamma} \sin (y-x)\right] \vartheta
\end{aligned}
$$

where $y=\eta k / \sqrt{3}$ is proportional to the scale factor, while $c_{i}\left(x^{i}\right)$ are two integration constants. As one may see from this expression, for $-1 \leq \gamma<0$ the $\vartheta$-dependent contribution to the density contrast decays with the scale factor so that only the standard contribution remains.
Thus, in this range of $\gamma$ the perturbations behave just as predicted by general relativity in both matter and radiation-dominated epochs.

Another case of interest is $\gamma=1$. This case is special because at $\gamma=1$ the $a$-dependence of the last term in eq. (26) disappears. In fact, one may show that in this case the dependence on $\vartheta$ cancels out in the density contrast, so that only the standard part of perturbations remains.

At other values of $\gamma$ the $\vartheta$-dependent contributions to perturbations grow in the radiation-dominated Universe.

\section{DISCUSSION}

To summarize, in the model of massive gravity described by the action (1) the cosmological perturbations contain two parts, the "normal" and the "anomalous" one. The first, normal part has the behavior identical to that found in the conventional general relativity. It is therefore in agreement with observations to the same extent as the latter. In particular, the "normal" part of the perturbations can describe successfully at least the linear stage of the structure formation. In fact, the predictions of the general relativity and the model (1) with only the "normal" perturbations present completely coincide in the linear regime, so that the two models are indistinguishable in this respect.

The second, "anomalous" part of perturbations is specific to the model of massive gravity with the action (1). These perturbations originate from the condensates of the scalar fields present in the model. This contribution to perturbations depends linearly on the unknown function of space coordinates $\vartheta\left(x^{i}\right)$ which enters as an integration constant. The value of this function cannot be determined within the model (1) and has to be specified as an initial conditions.

The behavior of the "anomalous" contributions to perturbations at different stages of the evolution of the Universe depends on the value of $\gamma$. At the matterdominated stage the anomalous perturbations grow not faster than the standard ones for $\gamma \geq 1$ and $\gamma<0$. In the radiation-dominated epoch this occurs at $-1 \leq \gamma<0$ and $\gamma=1$. Thus, at $-1 \leq \gamma<0$ and $\gamma=1$ the normal perturbations dominate at both radiation and matterdominated stages.

The appearance of the time-independent arbitrary function is not surprising. The same function $\vartheta\left(x^{i}\right)$ also enters the expression for the gravitational potential of an isolated massive body [12]. Its origin may be traced back to the existence of the scalar mode with the dispersion relation $\omega^{2}=0$ [11]. This mode is not dynamical in the model (1). However, the action (1) is the low-energy effective action, so one should expect the corrections containing higher-derivative terms to be present. In general, these corrections make $\vartheta$ a dynamical variable with the dispersion relation $\omega^{2}=\alpha p^{4}$, where $\alpha$ is a small coefficient. Therefore, $\vartheta$ becomes a slowly varying function 
of time. The slow evolution may drive $\vartheta$ to a particular value at the inflationary epoch and thus prepare the initial conditions for the radiation-dominated stage. If this initial value of $\vartheta$ is small, then the growth of the "anomalous" part of perturbations may become irrelevant and corresponding values of $\gamma$ phenomenologically acceptable. This question will be considered elsewhere.

\section{Acknowledgments}

The authors are grateful to S. Dubovsky for valuable discussions and reading of the manuscript. The work of M.B. is supported by the Belgian FRIA, Fond pour la Formation à la Recherche dans l'Industrie et dans l'Agronomie. The work of P.T. is supported by IISN, Belgian Science Policy (under contract IAP V/27).

\section{APPENDIX}

In these Appendix we provide some intermediate formulae skipped in the main text. In Appendix A we calculate the field equations for the standard FRW solution (3). In Appendix B we introduce the mass parameters and the relations between them. In Appendix we calculate the gauge-invarant linearized field equations.

\section{APPENDIX A: THE BACKGROUND}

For $X, V^{i}$ and $W^{i j}$ given by eq. (2), the following action describes a massive gravitational field

$$
\mathcal{S}=\int \mathrm{d}^{4} x \sqrt{-g}\left[-M_{P l}^{2} \mathcal{R}+\Lambda^{4} \mathcal{F}\left(X, V^{i}, W^{i j}, \ldots\right)\right],
$$

characterized by the ten Einstein equations $\mathcal{G}_{\mu \nu}=$ $M_{p l}^{-2}\left(\mathcal{T}_{\mu \nu}^{m}+\mathcal{T}_{\mu \nu}^{\phi}\right)$ and four Goldstone equations

$$
\begin{aligned}
0= & \partial_{\beta}\left\{\sqrt { - g } g ^ { \alpha \beta } \left[\frac{\partial \mathcal{F}}{\partial X} \delta_{\mu}^{0} \partial_{\alpha} \phi^{0}+\frac{\partial \mathcal{F}}{\partial W^{i j}}\left(\delta_{\mu}^{i} \partial_{\alpha} \phi^{j}\right.\right.\right. \\
& \left.-\frac{V^{j}}{X} \partial_{\alpha}\left(\phi^{0} \delta_{\mu}^{i}+\phi^{i} \delta_{\mu}^{0}\right)+\delta_{\mu}^{0} \frac{V^{i} V^{j}}{X^{2}} \partial_{\alpha} \phi^{0}\right) \\
& \left.\left.+\frac{1}{2} \frac{\partial \mathcal{F}}{\partial V^{i}} \partial_{\alpha}\left(\phi^{0} \delta_{\mu}^{i}+\phi^{i} \delta_{\mu}^{0}\right)\right]\right\} .
\end{aligned}
$$

Here $\mathcal{T}_{\mu \nu}^{m}$ is the energy-momentum tensor of the ordinary matter which has the standard form (8) while the energymomentum tensor of the Goldstone field is given by

$$
\begin{aligned}
\mathcal{T}_{\mu \nu}^{\phi}= & \Lambda^{4}\left\{-\frac{1}{2} g_{\mu \nu} \mathcal{F}+\frac{1}{2} \frac{\partial \mathcal{F}}{\partial V^{i}}\left(\partial_{\mu} \phi^{i} \partial_{\nu} \phi^{0}+\partial_{\mu} \phi^{0} \partial_{\nu} \phi^{i}\right)\right. \\
& +\left[\partial_{\mu} \phi^{i} \partial_{\nu} \phi^{j}-\frac{V^{j}}{X}\left(\partial_{\mu} \phi^{i} \partial_{\nu} \phi^{0}+\partial_{\mu} \phi^{0} \partial_{\nu} \phi^{i}\right)\right. \\
& \left.\left.+\frac{V^{i} V^{j}}{X^{2}} \partial_{\mu} \phi^{0} \partial_{\nu} \phi^{0}\right] \frac{\partial \mathcal{F}}{\partial W^{i j}}+\frac{\partial \mathcal{F}}{\partial X} \partial_{\mu} \phi^{0} \partial_{\nu} \phi^{0}\right\} .
\end{aligned}
$$

For the cosmological solutions given by eq. (3), $X=$ $a^{-2} \phi^{\prime 2}, V^{i}=0$ and $W^{i j}=-a^{-2} \delta^{i j}$. The 14 field equations reduce to the following three relations (prime stands for the derivative with respect to $\eta$ ),

$$
\begin{aligned}
3 \mathcal{H}^{2} & =\frac{a^{2}}{M_{p l}^{2}}\left(\rho_{m}+\rho_{\phi}+\rho_{\Lambda}\right), \\
2 \mathcal{H}^{\prime}+\mathcal{H}^{2} & =-\frac{a^{2}}{M_{p l}^{2}}\left(p_{m}+p_{\phi}+p_{\Lambda}\right), \\
0 & =\partial_{0}\left(a^{3} \mathcal{F}_{X} X^{1 / 2}\right)
\end{aligned}
$$

which can be solved for any given function $\mathcal{F}(a, X)$. The following notations were used

$$
\begin{array}{ll}
\rho_{\phi}=\Lambda^{4} X \mathcal{F}_{X}, & p_{\phi}=\Lambda^{4} W \mathcal{F}_{W}, \\
\rho_{\Lambda}=-\Lambda^{4} \mathcal{F} / 2, & p_{\Lambda}=\Lambda^{4} \mathcal{F} / 2 .
\end{array}
$$

For the model (1) characterized by a function $\mathcal{F}$ depending only on $Z^{i j}$ one has $p_{\phi}=-(3 \gamma)^{-1} \rho_{\phi}$.

\section{APPENDIX B: THE MASS PARAMETERS}

There are five mass parameters $m_{i}^{2}, i=0 \ldots 4$ defined by the following relations

$$
\begin{aligned}
m_{0}^{2} & =\frac{\Lambda^{4}}{M_{P l}^{2}}\left(X \mathcal{F}_{X}+2 X^{2} \mathcal{F}_{X X}\right) \\
m_{1}^{2} & =\frac{2 \Lambda^{4}}{M_{P l}^{2}}\left(-X \mathcal{F}_{X}-W \mathcal{F}_{W}+\frac{1}{2} X W \mathcal{F}_{V V}\right) \\
m_{2}^{2} & =\frac{2 \Lambda^{4}}{M_{P l}^{2}}\left(W \mathcal{F}_{W}-2 W^{2} \mathcal{F}_{W W 2}\right) \\
m_{3}^{2} & =\frac{\Lambda^{4}}{M_{P l}^{2}}\left(W \mathcal{F}_{W}+2 W^{2} \mathcal{F}_{W W 1}\right) \\
m_{4}^{2} & =-\frac{\Lambda^{4}}{M_{P l}^{2}}\left(X \mathcal{F}_{X}+2 X W \mathcal{F}_{X W}\right)
\end{aligned}
$$

where $W=-1 / 3 \delta_{i j} W^{i j}$ and where the first and second derivatives of the function $\mathcal{F}\left(X, V^{i}, W^{i j}, \ldots\right)$ are denoted as follows

$$
\begin{aligned}
\frac{\partial \mathcal{F}}{\partial X} & \equiv \mathcal{F}_{X}, \\
\frac{\partial^{2} \mathcal{F}}{\partial X^{2}} & \equiv \mathcal{F}_{X X}, \\
\frac{\partial^{2} \mathcal{F}}{\partial V^{i} V^{j}} & \equiv \mathcal{F}_{V V} \delta_{i j}, \\
\frac{\partial \mathcal{F}}{\partial W^{i j}} & \equiv \mathcal{F}_{W} \delta_{i j}, \\
\frac{\partial^{2} \mathcal{F}}{\partial X W^{i j}} & \equiv \mathcal{F}_{X W} \delta_{i j}, \\
\frac{\partial^{2} \mathcal{F}}{\partial W^{i j} W^{k l}} & \equiv \mathcal{F}_{W W 1} \delta_{i j} \delta_{k l}+\mathcal{F}_{W W 2}\left(\delta_{i k} \delta_{j l}+\delta_{i l} \delta_{j k}\right) .
\end{aligned}
$$


It is possible to relate the masses $m_{0}^{2}$ and $m_{4}^{2}$ by using the Goldstone equation for the background. This last gives

$$
m_{0}^{2}\left(\frac{\phi^{\prime \prime}}{\phi^{\prime}}-\mathcal{H}\right)=3 \mathcal{H} m_{4}^{2}
$$

For the model characterized by the function $\mathcal{F}=$ $\mathcal{F}\left(Z^{i j}\right)$, it is straightforward to show that

$$
\begin{aligned}
m_{0}^{2}= & \frac{\Lambda^{4}}{\mathcal{M}_{p l}^{2}} \gamma\left[3(1-2 \gamma) Z \mathcal{F}_{Z}\right. \\
& \left.+6 \gamma Z^{2}\left(3 \mathcal{F}_{Z Z 1}+2 \mathcal{F}_{Z Z 2}\right)\right], \\
m_{1}^{2}= & \frac{2 \Lambda^{4}}{M_{p l}^{2}}(3 \gamma-1) Z \mathcal{F}_{Z}, \\
m_{2}^{2}= & \frac{2 \Lambda^{4}}{\mathcal{M}_{p l}^{2}}\left(Z \mathcal{F}_{Z}-2 Z^{2} \mathcal{F}_{Z Z 2}\right), \\
m_{3}^{2}= & \frac{\Lambda^{4}}{\mathcal{M}_{p l}^{2}}\left(Z \mathcal{F}_{Z}+2 Z^{2} \mathcal{F}_{Z Z 1}\right), \\
m_{4}^{2}= & \frac{\Lambda^{4}}{\mathcal{M}_{p l}^{2}} \gamma\left[Z \mathcal{F}_{Z}+2 Z^{2}\left(3 \mathcal{F}_{Z Z 1}+2 \mathcal{F}_{Z Z 2}\right)\right],
\end{aligned}
$$

where

$$
\begin{aligned}
\frac{\partial \mathcal{F}}{\partial Z^{i j}} & \equiv \mathcal{F}_{Z} \delta_{i j}, \\
\frac{\partial^{2} \mathcal{F}}{\partial Z^{i j} Z^{k l}} & \equiv \mathcal{F}_{Z Z 1} \delta_{i j} \delta_{k l}+\mathcal{F}_{Z Z 2}\left(\delta_{i k} \delta_{j l}+\delta_{i l} \delta_{j k}\right) .
\end{aligned}
$$

For this particular case, $-3 \gamma p_{\phi}=\rho_{\phi}$ and the following relations hold

$$
\left\{\begin{array}{l}
m_{0}^{2}=3 \gamma\left(m_{4}^{2}-m_{1}^{2} / 2\right) \\
m_{4}^{2}=\gamma\left(3 m_{3}^{2}-m_{2}^{2}\right) \\
m_{1}^{2}=2(3 \gamma-1) p_{\phi}
\end{array}\right.
$$

\section{APPENDIX C: THE LINEARIZED EQUATIONS}

Small perturbations on the cosmological background can be expressed trough eq. (6-7). The tensor field $h_{i j}$ and the vector field $\zeta_{i}$ are gauge-invariant, while the other two gauge-invariant vector fields and seven gaugeinvariant scalar fields are introduced trough eq. (9-10). With all these definitions, the linearized part of the Ein- stein tensor is given by

$$
\begin{aligned}
\delta \mathcal{G}_{00}= & 2 \partial_{i}^{2} \Psi-6 \mathcal{H} \psi^{\prime}, \\
\delta \mathcal{G}_{i 0}= & \partial_{i}\left[2\left(\mathcal{H} \varphi+\psi^{\prime}\right)+\left(2 \mathcal{H}^{\prime}+\mathcal{H}^{2}\right) B\right]+\frac{1}{2} \partial_{i}^{2} \varpi_{i} \\
& +v_{i}\left(2 \mathcal{H}^{\prime}+\mathcal{H}^{2}\right), \\
\delta \mathcal{G}_{i j}= & -\delta_{i j}\left[2(\varphi+\psi)\left(2 \mathcal{H}^{2}+\mathcal{H}^{\prime}\right)-2 \mathcal{H}(2 \psi+\varphi)^{\prime}\right. \\
& \left.-2 \psi^{\prime \prime}+\partial_{i}^{2}(\Psi-\Phi)-6(\psi+\varphi)\left(\mathcal{H}^{2}+\mathcal{H}^{\prime}\right)\right] \\
& +\partial_{i} \partial_{j}\left[\Psi-\Phi-2\left(2 \mathcal{H}^{\prime}+\mathcal{H}^{2}\right) E\right]-\mathcal{H} h_{i j}^{\prime} \\
& +\frac{1}{2}\left(\partial_{i}^{2} h_{i j}-h_{i j}^{\prime \prime}\right)+\left(2 \mathcal{H}^{\prime}+\mathcal{H}^{2}\right) h_{i j} \\
& +\mathcal{H}\left(\partial_{i} \varpi_{j}+\partial_{j} \varpi_{i}\right)+\frac{1}{2}\left(\partial_{i} \varpi_{j}^{\prime}+\partial_{j} \varpi_{i}^{\prime}\right) \\
& -\left(\mathcal{H}^{2}+2 \mathcal{H}^{\prime}\right)\left(\partial_{i} F_{j}+\partial_{j} F_{i}\right) .
\end{aligned}
$$

The linearized energy-momentum tensor of the ordinary matter fields is given by eq. (8), while the linearized energy momentum tensor of the Goldstone fields takes the form

$$
\begin{aligned}
\delta \mathcal{T}_{00}^{\phi}= & a^{2} M_{P l}^{2}\left[\left(2 \rho_{\phi}+2 \rho_{\Lambda}-m_{0}^{2}\right) \varphi+m_{0}^{2} \xi^{0 \prime} / \phi^{\prime}\right. \\
& \left.+\left(\rho_{\phi}+p_{\phi}+m_{4}^{2}\right)\left(\partial_{i}^{2} \Xi+3 \psi\right)\right], \\
\delta \mathcal{T}_{0 i}^{\phi}= & {\left[\frac{\rho_{\phi}+p_{\phi}}{M_{P l}^{2}}\left(\xi_{i}+\partial_{i} \xi\right)^{\prime}+\frac{\rho_{\phi}+\rho_{\Lambda}}{M_{P l}^{2}}\left(v_{i}+\partial_{i} B\right)\right.} \\
& \left.+\frac{m_{1}^{2}}{2}\left(v_{i}+\partial_{i} \mathcal{B}+\xi_{i}^{\prime}+\partial_{i} \Xi^{\prime}\right)\right] a^{2} M_{P l}^{2}, \\
\delta \mathcal{T}_{i j}^{\phi}= & a^{2} M_{P l}^{2}\left[\frac{1}{2} m_{2}^{2}\left(\partial_{j} \xi_{i}+\partial_{i} \xi_{j}+2 \partial_{i} \partial_{j} \xi\right)\right. \\
& -m_{3}^{2} \delta_{i j}\left(3 \psi+\partial_{i}^{2} \Xi\right)+\delta_{i j} m_{4}^{2}\left(\varphi-\xi^{0 \prime} / \phi^{\prime}\right) \\
& +\left(\frac{1}{2} m_{2}^{2}-\frac{p_{\phi}+p_{\Lambda}}{M_{P l}^{2}}\right)\left(2 \psi \delta_{i j}-\partial_{i} F_{j}-\partial_{j} F_{i}\right. \\
& \left.\left.-2 \partial_{i} \partial_{j} E+h_{i j}\right)\right] .
\end{aligned}
$$

These relations allow to write the linearized Einstein field equations for the massive gravitational field. They consist of one tensor equation

$$
0=h_{i j}^{\prime \prime}-\partial_{i}^{2} h_{i j}+2 \mathcal{H} h_{i j}^{\prime}+a^{2} m_{2}^{2} h_{i j}
$$

two vector equations

$$
0=\varpi_{i}^{\prime}+2 \mathcal{H} \varpi_{i}-a^{2} m_{2}^{2} \sigma_{i}
$$

$$
\begin{aligned}
0= & a^{-2} \partial_{i}^{2} \varpi_{i}-\left(m_{1}^{2}+2 \frac{\rho_{\phi}+p_{\phi}}{M_{P l}^{2}}\right)\left(\varpi_{i}+\sigma_{i}^{\prime}\right) \\
& -2 \frac{\rho_{m}}{M_{P l}^{2}}\left(1+\omega_{m}\right) \zeta_{i},
\end{aligned}
$$


and four scalar equations

$$
\begin{aligned}
0= & -2 \partial_{j}^{2} \Psi+6 \mathcal{H}\left(\mathcal{H} \Phi+\Psi^{\prime}\right)-a^{2} m_{0}^{2}\left[\frac{\phi^{\prime \prime}}{\phi^{\prime}} \mathcal{B}+\mathcal{B}^{\prime}+\Phi\right] \\
& +a^{2}\left(\frac{\rho_{\phi}+p_{\phi}}{M_{P l}^{2}}+m_{4}^{2}\right)\left(\partial_{j}^{2} \Xi+3 \Psi\right)+a^{2} \frac{\rho_{m}}{M_{P l}^{2}} \delta_{\rho}, \\
0= & 2 \partial_{i}\left(\Psi^{\prime}+\mathcal{H} \Phi\right)+a^{2} \partial_{i}\left[-\frac{m_{1}^{2}}{2}\left(\mathcal{B}+\Xi^{\prime}\right)\right. \\
& \left.-\frac{\rho_{\phi}+p_{\phi}}{M_{P l}^{2}} \Xi^{\prime}-\frac{\rho_{m}}{M_{P l}^{2}}\left(1+w_{\omega}\right) \delta_{\zeta}\right], \\
0 & \partial_{i} \partial_{j}\left(\Phi-\Psi+a^{2} m_{2}^{2} \Xi\right), \\
0 & -2 \Psi^{\prime \prime}-2 \Phi\left(\mathcal{H}^{2}+2 \mathcal{H}^{\prime}\right)+\partial_{j}^{2}(\Psi-\Phi) \\
& -2 \mathcal{H}(2 \Psi+\Phi)^{\prime}+a^{2} \frac{p_{m}}{M_{P l}^{2}} \delta_{p}-a^{2} m_{3}^{2} \partial_{j}^{2} \Xi \\
& +a^{2} m_{4}^{2}\left(\Phi+\frac{\phi^{\prime \prime}}{\phi^{\prime}} \mathcal{B}+\mathcal{B}^{\prime}\right)+a^{2}\left(m_{2}^{2}-3 m_{3}^{2}\right) \Psi .
\end{aligned}
$$

The linearized Goldstone equations give one vector equa- tion

$$
0=a^{-4} \partial_{0}\left[a^{4}\left(m_{1}^{2}+2 \frac{\rho_{\phi}+p_{\phi}}{M_{P l}^{2}}\right)\left(\varpi_{i}+\sigma_{i}^{\prime}\right)\right]-m_{2}^{2} \partial_{j}^{2} \sigma_{i},
$$

and two scalar equations

$$
\begin{aligned}
0= & \partial_{0}\left[\frac{a^{4}}{\phi^{\prime}}\left(m_{0}^{2}\left(\frac{\phi^{\prime \prime}}{\phi^{\prime}} \mathcal{B}+\mathcal{B}^{\prime}+\Phi\right)-m_{4}^{2}\left(3 \Psi+\partial_{i}^{2} \Xi\right)\right)\right] \\
& +\frac{a^{4}}{2 \phi^{\prime}} m_{1}^{2} \partial_{i}^{2}\left(\mathcal{B}+\Xi^{\prime}\right), \\
0= & \partial_{i} \partial_{0}\left\{a^{4}\left[\left(\frac{m_{1}^{2}}{2}+\frac{\rho_{\phi}}{M_{P l}^{2}}\right)\left(\mathcal{B}+\Xi^{\prime}\right)+\frac{p_{\phi}}{M_{P l}^{2}} \Xi^{\prime}\right]\right\} \\
& +a^{4} \partial_{i}\left[\left(m_{3}^{2}-m_{2}^{2}\right) \partial_{j}^{2} \Xi-\left(m_{4}^{2}+\frac{\rho_{\phi}+p_{\phi}}{M_{P l}^{2}}\right) \Phi\right. \\
& \left.+\left(3 m_{3}^{2}-m_{2}^{2}\right) \Psi-\left(m_{4}^{2}+\frac{\rho_{\phi}}{M_{P l}^{2}}\right)\left(\frac{\phi^{\prime \prime}}{\phi^{\prime}} \mathcal{B}+\mathcal{B}^{\prime}\right)\right] .
\end{aligned}
$$

[1] D. N. Spergel et al. (WMAP), Astrophys. J. Suppl. 170, 377 (2007), astro-ph/0603449.

[2] M. Milgrom, Astrophys. J. 270, 371 (1983).

[3] J. D. Bekenstein, Phys. Rev. D70, 083509 (2004), astroph/0403694.

[4] R. Gregory, V. A. Rubakov, and S. M. Sibiryakov, Phys. Rev. Lett. 84, 5928 (2000), hep-th/0002072.

[5] G. R. Dvali, G. Gabadadze, and M. Porrati, Phys. Lett. B485, 208 (2000), hep-th/0005016.

[6] S. M. Carroll, V. Duvvuri, M. Trodden, and M. S. Turner, Phys. Rev. D70, 043528 (2004), astro-ph/0306438.

[7] I. I. Kogan, S. Mouslopoulos, and A. Papazoglou, Phys. Lett. B501, 140 (2001), hep-th/0011141.

[8] T. Damour and I. I. Kogan, Phys. Rev. D66, 104024 (2002), hep-th/0206042.

[9] N. Arkani-Hamed, H.-C. Cheng, M. A. Luty, and S. Mukohyama, JHEP 05, 074 (2004), hep-th/0312099.

[10] V. A. Rubakov (2004), hep-th/0407104.
[11] S. L. Dubovsky, JHEP 10, 076 (2004), hep-th/0409124.

[12] S. L. Dubovsky, P. G. Tinyakov, and I. I. Tkachev, Phys. Rev. Lett. 94, 181102 (2005), hep-th/0411158.

[13] S. L. Dubovsky, P. G. Tinyakov, and I. I. Tkachev, Phys. Rev. D72, 084011 (2005), hep-th/0504067.

[14] V. F. Mukhanov, H. A. Feldman, and R. H. Brandenberger, Phys. Rept. 215, 203 (1992).

[15] To be precise, the vacuum solution reads $\phi^{0}=a t, \phi^{i}=$ $b x^{i}$, where $a$ and $b$ are two constants whose value is set by the requirement that the energy-momentum of the scalar fields in the Minkowski space vanishes. We assume in what follows that the function $\mathcal{F}$ is such that $a=b=\Lambda^{2}$.

[16] This feature is obviously a consequence of the breakdown of the Lorentz invariance.

[17] This ansatz cannot be directly generalized to the cases of open and closed Universe [11]. It is not known whether the model admits cosmological solutions of these types. 